\title{
Spectroscopy analysis for simultaneous determination of lycopene and $\beta$-carotene in fungal biomass of Blakeslea trispora*
}

\author{
Iaroslav M. Soroka1, Valeriy G. Narushin ${ }^{2 \varpi}$, Yuriy D. Turiyansky ${ }^{1}$ and Alexey A. Tyurenkov ${ }^{1}$ \\ IVitan Group, Vitan Ltd., Dneprovsky, Dnepropetrovsk Region, Ukraine; ${ }^{2}$ Vitan Group, Vita-Market Ltd., Zaporozhye, Ukraine
}

\begin{abstract}
Blakeslea trispora is a good alternative source for producing such carotenoids as lycopene and $\beta$-carotene. The objective of this research was to elaborate a method for the simultaneous determination of lycopene and $\beta$-carotene in Blakeslea trispora products using a usual UV-vis spectrophotometer. The standard solutions of the mixture of different concentrations of $\beta$-carotene and lycopene were measured with the UV-vis method and correlation formula for the extinction coefficients of $1 \%$ standard solution of lycopene in the solvent (hexane) and the ratios of the optical densities at the character peaks of 470 and $502 \mathrm{~nm}$ was elaborated. This gives a possibility to calculate the concentrations of lycopene and $\beta$-carotene in the mixture. The prediction quality of the UV-vis method was sufficient and the obtained results were very close to the ones, being measured with the HPLC technique. The proposed method can be used for both routine industrial work and academic research, providing the rapid analysis for simultaneous measurements of lycopene and $\beta$-carotene.
\end{abstract}

Key words: lycopene; $\beta$-carotene; UV-vis analysis

Received: 28 September, 2011; accepted: 01 March, 2012; available on-line: 17 March, 2012

\section{INTRODUCTION}

Blakeslea trispora is a good alternative source for producing such carotenoids as lycopene and $\beta$-carotene. Blakeslea trispora is a heterothallic Zygomycota with two mating types (termed plus and minus). These two opposite mating types produce zygophores, which grow toward each other and produce progametangia on their tips. Septation in the progametangia leads to the production of terminal gametangia, which fuse to form the zygospores, which are responsible for the biosynthesis of the pigment (Mantzouridou et al., 2002). $\beta$-carotene results from the formation of $\beta$-rings at both ends of a linear precursor, lycopene, as shown by genetic (Mehta \& Cerda-Olmedo, 1995) and chemical (Mehta \& Cerda-Olmedo, 1999) inhibition of this reaction. It was shown by various studies (Mehta et al., 2003; Mantzouridou \& Tsimidou, 2007; Pegklidou et al., 2008; Choudhari et al., 2008) that both carotenoids ( $\beta$-carotene and lycopene) are presented together in the fungal biomass of Blakeslea trispora, even in a case of inhibition of the $\beta$-carotene synthesis.

Both industrial manufacturing of the Blakeslea trispora fungal biomass, as also different research investigation in this field require an accurate, simple, and fast method for evaluation of lycopene and $\beta$-carotene in the final and intermediate products. The basic methods of analyzing lycopene and/or $\beta$-carotene by UV-vis spectrophotom- eter is described in Britton et al. (2004). But the methods are good when there is only one carotenoid in the sample. When the both carotenoids are presented together, the problem in the accurate determination of each of them appears to be under incorrect spectra shapes which are resulted by overlapping of the peaks that correspond to either lycopene or $\beta$-carotene. Currently, separation and quantification of the mix carotenoids estimate with HPLC (Mantzouridou \& Tsimidou, 2007; Emenhiser et al., 1996; Cserhati et al., 2000; Oliver.\& Palou, 2000; Echavarri-Erasun \& Johnson, 2002; Xu et al., 2006). Although reliable and accurate, the HPLC method is timeconsuming and requires extensive sample preparation and the use and disposal of hazardous organic solvents (Nardo et al., 2009). Some alternative methods for simultaneous determination of lycopene and $\beta$-carotene have been proposed. These include infrared spectroscopy (Nardo et al., 2009; Pedro \& Ferreira, 2005; Baranska et al., 2006) and resonance Raman spectroscopy (Ermakov et al., 2004). The proposed methods require some additional techniques besides the UV-vis spectrophotometer, and this may cause some restrictions in introducing such method for the practical use.

The objective of this research was to elaborate a method for the simultaneous determination of lycopene and $\beta$-carotene using a usual UV-vis spectrophotometer.

\section{MATERIALS AND METHODS}

Standard Samples. $\beta$-carotene oil suspension 30\% (CaroPure ${ }^{\circledR}$ ) from Blakeslea trispora was received as a gift from DSM Nutritional Products (Basel, Switzerland). Lycopene oil suspension 6\% $\left(\right.$ LycoNat $\left.^{\circledR}\right)$ from Blakeslea trispora was received as a gift from Vitatene (Leon, Spain).

The sample of $0.00205 \mathrm{~g}$ of the $30 \% \beta$-carotene oil suspension was dissolved in $100 \mathrm{ml}$ of hexane $\left(V_{\mathrm{car}}\right)$. The sample of $0.0126 \mathrm{~g}$ of the $6 \%$ lycopene oil suspension was dissolved in $100 \mathrm{ml}$ of hexane $\left(V_{\text {lvc }}\right)$. The following solutions were prepared by mixing $V_{\text {car }}$ and $V_{\text {lyc }}$ in different combinations:

$$
\begin{aligned}
& 1 \mathrm{ml} V_{\text {lyc }}+0 \mathrm{ml} V_{\text {car }} \\
& 1 \mathrm{ml} V_{\text {lyc }}+0.2 \mathrm{ml} V_{\text {car }} \\
& 1 \mathrm{ml} V_{\text {lyc }}+0.4 \mathrm{ml} V_{\text {car }} \\
& 1 \mathrm{ml} V_{\text {lyc }}+0.6 \mathrm{ml} V_{\text {car }}
\end{aligned}
$$

e-mail: narushin@vitan.ru

*Presented at the 16th International Symposium on Carotenoids, 17-22 July, 2011, Kraków, Poland 
$1 \mathrm{ml} V_{\text {lyc }}+0.8 \mathrm{ml} V_{\mathrm{car}}$
$1 \mathrm{ml} V_{\text {lyc }}+1 \mathrm{ml} V_{\mathrm{car}}$
$1 \mathrm{ml} V_{\text {lyc }}+2 \mathrm{ml} V_{\text {car }}$
$1 \mathrm{ml} V_{\text {lyc }}+3 \mathrm{ml} V_{\mathrm{car}}$
$1 \mathrm{ml} V_{\text {lyc }}+4 \mathrm{ml} V_{\mathrm{car}}$
$1 \mathrm{ml} V_{\text {lyc }}+5 \mathrm{ml} V_{\mathrm{car}}$
$0 \mathrm{ml} V_{\text {lyc }}+1 \mathrm{ml} V_{\mathrm{car}}$

Each concentration was measured with a UV-vis spectrophotometer at a range of 400-520 $\mathrm{nm}$.

Blakeslea trispora samples. The Blakeslea trispora cells were grown in a $10 \mathrm{~m}^{3}$ fermenter according to (Patent, 2008). The samples of the fungal biomass of Blakeslea trispora have been analyzed with a UV-vis spectroscopy and with HPLC.

UV-Vis spectroscopy analysis. A Hedios Gamma UV visible spectrophotometer (Thermo Spectronic, Cambridge, UK) was used for the analyses. The sample of the fungal biomass (1-3 $\mathrm{mg}$ ) was weighted using a balance with an accuracy of up to the fifth decimal and then put into a round-bottom test tube. Hexane in a quantity of $5 \mathrm{ml}$ was added to the test tube with the sample and the biomass was thoroughly ground using a glass stick until the complete discoloration of the biomass particles. The content of the test tube was put into a $50 \mathrm{ml}$ flask. The test tube was washing several times with hexane until the solvent became uncolored. An additional quantity of pure hexane was added to reach $35-40 \mathrm{ml}$ of the obtained solution in the flask. The flask was closed with a cork cap. The solution was mixed by means of turning the flask over and then put into a dark place for 30-40 minutes at a room temperature until the biomass sample was absolutely uncolored. After settling,

Table 1 . The concentrations of pure lycopene and $\beta$-carotene in the standard samples.

\begin{tabular}{|c|c|c|}
\hline Standard sample & $\begin{array}{l}\text { Concentration of } \\
\text { lycopene in the } \\
\text { standard sample } \\
\text { in } \%\end{array}$ & $\begin{array}{l}\text { Concentration of } \\
\beta \text {-carotene in the } \\
\text { standard sample } \\
\text { in } \%\end{array}$ \\
\hline $1 \mathrm{ml} V_{\text {lyc }}+0 \mathrm{ml} V_{\text {car }}$ & 6.0 & 0 \\
\hline $1 \mathrm{ml} V_{\text {lyc }}+0.2 \mathrm{ml} V_{\text {car }}$ & 5.8 & 0.9 \\
\hline $1 \mathrm{ml} V_{\text {lyc }}+0.4 \mathrm{ml} V_{\text {car }}$ & 5.7 & 1.8 \\
\hline $1 \mathrm{ml} V_{\text {lyc }}+0.6 \mathrm{ml} V_{\text {car }}$ & 5.5 & 2.7 \\
\hline $1 \mathrm{ml} V_{\text {lyc }}+0.8 \mathrm{ml} V_{\text {car }}$ & 5.3 & 3.5 \\
\hline $1 \mathrm{ml} V_{\text {lyc }}+1 \mathrm{ml} V_{\text {car }}$ & 5.2 & 4.2 \\
\hline $1 \mathrm{ml} V_{\text {lyc }}+2 \mathrm{ml} V_{\text {car }}$ & 4.6 & 7.4 \\
\hline $1 \mathrm{ml} V_{\text {lyc }}+3 \mathrm{ml} V_{\text {car }}$ & 4.0 & 9.8 \\
\hline $1 \mathrm{ml} V_{\text {lyc }}+4 \mathrm{ml} V_{\text {car }}$ & 3.6 & 11.8 \\
\hline $1 \mathrm{ml} V_{\text {lyc }}+5 \mathrm{ml} V_{\text {car }}$ & 3.3 & 13.5 \\
\hline $0 \mathrm{ml} V_{\text {lyc }}+1 \mathrm{ml} V_{\text {car }}$ & 0 & 30.0 \\
\hline
\end{tabular}

hexane was added to the flask up to the $50 \mathrm{ml}$ quantity of the solution and mixed again several times by turning the flask over. After settling the particles of the biomass, the clear carotenoid-hexane solution was taken for the spectrophotometric measurements. Spectra were collected in the range of 400-520 $\mathrm{nm}$ and were displayed using local control software.

High-Performance Liquid Chromatography (HPLC) analysis. HPLC (Shimadzu Corp., Japan) was used as a reference method. The reverse-phase HPLC system was equipped with a photodiode array detector Waters 490 (USA). A column $\mathrm{p} / \mathrm{n}$ 70103-052130 s/n 0143831X (Thermo Electron Corp., USA) was used. Methanol:Tetrahydrofurane $(9: 1)$ was used as a fluid phase.

The sample preparation was similar to the spectroscopy analysis. The carotenoid-hexane solution was measured according to methods (Lycopene from Blakeslea trispora, 2006; $\beta$-carotene from Blakeslea trispora, 2003).

Calculations. All mathematical calculations have been undertaken using a computer softwasre package STATISTICA (1999).

\section{RESULTS AND DISCUSSION}

\section{Spectral analysis}

The concentrations of pure lycopene and $\beta$-carotene in the standard samples are shown in Table 1.

The concentrations of pure lycopene and $\beta$-carotene in the standard samples have been calculated using the formulae from Britton et al. (2004):

$$
C_{l y c}=\frac{D_{470} \cdot V}{m \cdot l \cdot A_{l y c-t a b}^{1 p^{106}}}
$$

in which $C_{\text {lyc }}$ is the percentage of lycopene in $\% ; D_{470}$ is the optical density of the investigated solution at the wavelength of $470 \mathrm{~nm}$; $V$ is the solvent (hexane) volume, being spent for the preparation of the investigated solution in $\mathrm{ml} ; m$ is the weight of the sample in $\mathrm{g} ; l$ is the thickness of the cuvette for the optical density measurements in cm; $A^{1 \%}{ }_{\text {lyc.tab }}$ is the table extinction coefficient of the $1 \%$ solution of pure lycopene, equals to 3450.

$$
C_{\beta-c a r}=\frac{D_{450} \cdot V}{m \cdot l \cdot A_{\beta-c a r-t a b}^{1 \%}}
$$

in which $C_{\beta-\text { cat }}$ is the percentage of $\beta$-carotene in $\% ; D_{450}$ is the optical density of the investigated solution at the wavelength of $450 \mathrm{~nm}$; $V$ is the solvent (hexane) volume, being spent for the preparation of the investigated solution in $\mathrm{ml} ; m$ is the weight of the sample in $\mathrm{g} ; l$ is the thickness of the cuvette for the optical density measurements in $\mathrm{cm} ; A^{1 \%}{ }_{\beta \text {-car-tab }}$ is the table extinction coefficient of the $1 \%$ solution of pure $\beta$-carotene, equals to 2590.

The absorptions of $\beta$-carotene and lycopene are quite similar, thus during the spectroscopic measurements of the mixture of these carotenoids the imposition of their spectra is taking place. Therefore, the spectrum of the mixed carotenoids changes its shape depending on the actual quantity of $\beta$-carotene and lycopene in the investigating mixture (Fig. 1). 


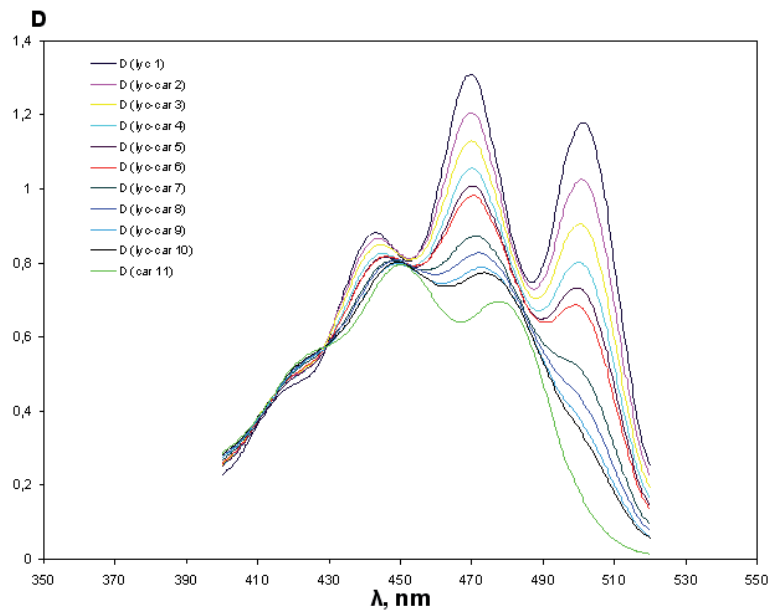

Figure 1. UV-vis spectra for the standard samples of the lycopene and $\beta$-carotene mixture.

The error comes from the inconstancy of the extinction coefficients $A^{1 \%}$. Thus to evaluate the percentage of lycopene in the sample which also contains $\beta$-carotene, the extinction coefficient of $1 \%$ solution of lycopene in the solvent (hexane) should be determined. It was found that besides the various extinction coefficients $A^{1 \%}$, the spectra of the carotenoid mixture have various shapes at different ratios of their concentrations, and each spectrum has quite expressed peaks at the wavelengths of $\lambda=470 \mathrm{~nm}\left(D_{470}\right)$ and $\lambda=502\left(D_{502}\right)$. The meanings of the extinction coefficient for lycopene $A^{1 \%}{ }_{\text {lyc }}$ were calculated using the Eqns (1) and the known concentrations of lycopene from the Table 1 . The data of extinction coefficients $A^{1 \%}$ and the spectral data of the optical densities $D_{470}$ and $D_{502}$ are shown in Table 2 .

It was found that the values of the extinction coefficients $\left(A^{1 \%}\right)$ are not constant and depended on the ratios of the optical densities $D_{470}: D_{502}$ (Fig. 2).

The relationship was expressed with the following empirical formula:

$$
\begin{aligned}
& A_{l y c}^{1 \%}=\left(-1.79+11.306 \cdot \frac{D_{470}}{D_{502}}\right. \\
& \left.0.061 \cdot\left(\frac{D_{470}}{D_{502}}\right)^{2}\right) \cdot e^{\left(5.696-0.09 \cdot\left(\frac{D_{470}}{D_{502}}\right)+0.147 \cdot\left(\frac{D_{470}}{D_{502}}\right)^{2}\right)}
\end{aligned}
$$

with $\mathrm{R}^{2}=0.998$, in which $A^{1 \%}{ }_{\text {lyc }}$ is the extinction coefficient of a $1 \%$ lycopene solution; $D_{470}$ is the optical density of the spectrum at $\lambda=470 \mathrm{~nm} ; D_{502}$ is the optical density of the spectrum at $\lambda=502 \mathrm{~nm}$.

Substituting the calculated from Eqn. (3) meaning of $A^{1 \%}{ }_{\text {lyc }}$ into Eqn. (1) allows us to determine the concentration of lycopene in the carotenoid mixture.

For any point of the measured spectra, the optical density of $\beta$-carotene can be calculated as a difference between the optical density of the mixed carotenoids and the optical density of lycopene in the same spectrum point (Peshkova \& Gromova, 1965). If to choose in our case a spectrum point, which corresponds to the wavelength of $470 \mathrm{~nm}$, the optical density for $\beta$-carotene $\left(D_{\beta \text {-car }}\right)$ is calculated as follows:

$D_{\beta \text {-car }}=D_{470}-D_{\text {lyc }}$

in which $D_{\beta \text {-car }}$ is the optical density for $\beta$-carotene at the wavelength of $470 \mathrm{~nm} ; D_{470}$ is the optical density of the

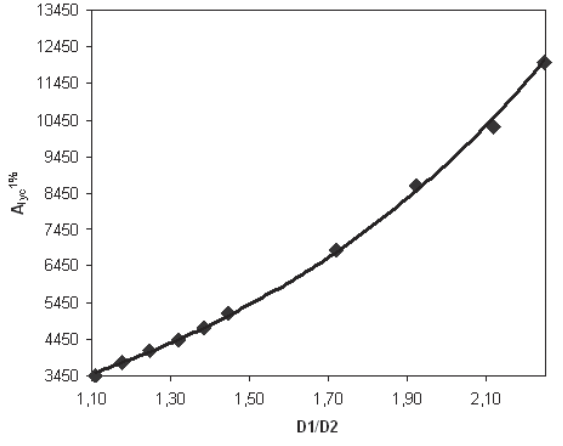

Figure 2. The correlation curve of the extinction coefficients $\left(A^{1 \% / y c}\right)$ and the ratios of the optical densities $D_{470}: D_{502}$.

mixed carotenoids in the mixture at the wavelength of $470 \mathrm{~nm} ; D_{\mathrm{lyc}}$ is the optical density of pure lycopene at the wavelength of $470 \mathrm{~nm}$.

The optical density of lycopene at the wavelength of $470 \mathrm{~nm} D_{\text {lyc }}$ is determined from Eqn. (1) using the known data of the lycopene concentration in the carotenoid mixture:

$D_{l y c}=\frac{C_{l y c} \cdot m \cdot l \cdot A_{l y c}^{1 \%}}{V}$

in which $D_{\mathrm{lyc}}$ is the optical density of pure lycopene in the carotenoid mixture; $C_{\mathrm{lyc}}$ is the known percentage of lycopene in the carotenoid mixture in $\% ; m$ is the weight of the sample in the carotenoid mixture in $g ; l$ is the thickness of the cuvette for the optical density measurements in $\mathrm{cm} ; A^{1 \%}$ iyc is the extinction coefficient of the $1 \%$ solution of pure lycopene in hexane at the wavelength of $470 \mathrm{~nm}$ being calculated by Eqn. (3); $V$ is the volume of the solvent (hexane), being spent for the preparation of the solution during the analysis in $\mathrm{ml}$.

To calculate finally the concentration of $\beta$-carotene in the carotenoid mixture by Eqn. (2), we need to know the extinction coefficient of the $1 \% \beta$-carotene solution $\left(A^{1 \%}{ }_{\beta \text {-car }}\right)$ in the spectrum point equals to $470 \mathrm{~nm}$ which is differed from the table meaning of 2590 for the wavelength of $450 \mathrm{~nm}$ being used by Britton et al. (2004). This is calculated from Eqn. (2) substituting the known concentration of $\beta$-carotene in the carotenoid mixture

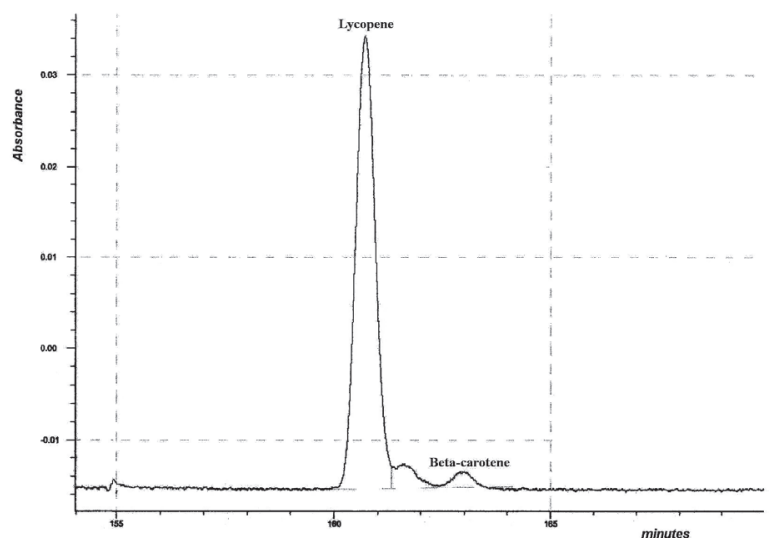

Figure 3. HPLC chromatogram of measuring carotenoid biomass of Blakeslea trispora. 
Table 2. The data of the optical densities for lycopene and $\beta$-carotene in the standard samples

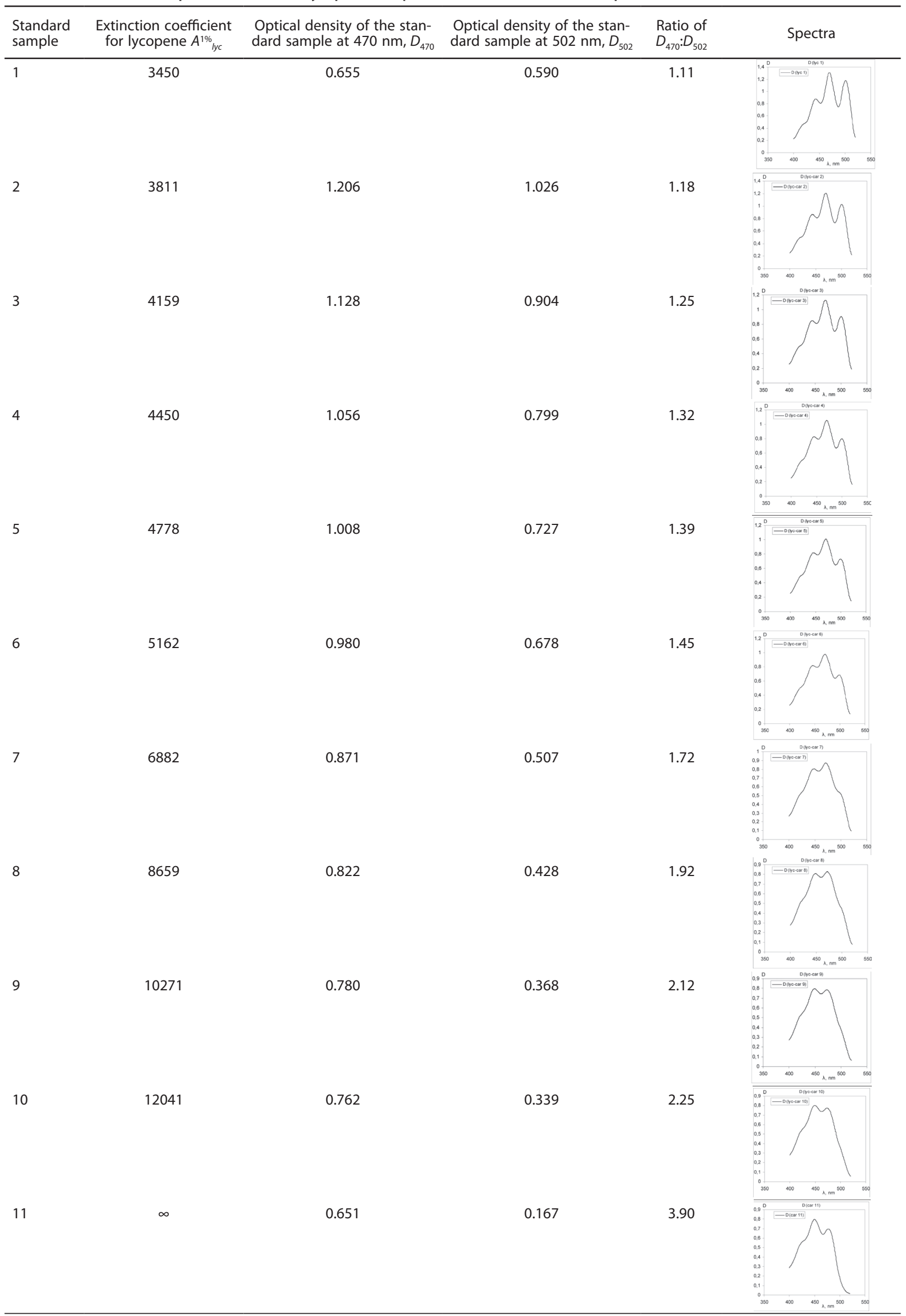


Table 3. The results of the measurements of lycopene and $\beta$-carotene in the fungal biomass of Blakeslea trispora using the UV-vis and HPLC methods.

\begin{tabular}{|c|c|c|c|c|}
\hline $\begin{array}{l}\text { Biomass } \\
\text { sample }\end{array}$ & $\begin{array}{l}\text { The concentration of } \\
\text { lycopene in the biomass } \\
\text { determined with UV-vis } \\
\text { method, \% }\end{array}$ & $\begin{array}{l}\text { The concentration of lyco- } \\
\text { pene in the biomass deter- } \\
\text { mined with HPLC method, \% }\end{array}$ & $\begin{array}{l}\text { The concentration of } \\
\beta \text {-carotene in the biomass } \\
\text { determined with UV-vis me- } \\
\text { thod, } \%\end{array}$ & $\begin{array}{l}\text { The concentration of } \\
\beta \text {-carotene in the biomass } \\
\text { determined with HPLC me- } \\
\text { thod, } \%\end{array}$ \\
\hline 1 & 5.3 & 5.2 & 0.7 & 0.7 \\
\hline 2 & 5.9 & 6.0 & 1.5 & 1.6 \\
\hline 3 & 5.3 & 5.4 & 1.2 & 1.2 \\
\hline 4 & 5.9 & 6.0 & 1.7 & 1.7 \\
\hline 5 & 5.9 & 5.9 & 1.1 & 1.2 \\
\hline 6 & 6.3 & 6.4 & 1.6 & 1.6 \\
\hline 7 & 6.0 & 6.0 & 0.9 & 1.0 \\
\hline 8 & 6.4 & 6.5 & 0.4 & 0.4 \\
\hline
\end{tabular}

corresponding to the spectrum point equals to $470 \mathrm{~nm}$. The result was found to be equal to 2116.

Calculating the optical density for $\beta$-carotene at the wavelength of $470 \mathrm{~nm}$ and knowing the extinction coefficient of the $1 \%$ solution of pure $\beta$-carotene in hexane $\left(A^{1 \%}{ }_{\beta \text {-car }}=2116\right)$ at the wavelength of $470 \mathrm{~nm}$, it is possible to determine the actual content of $\beta$-carotene in the carotenoid mixture.

\section{HPLC analysis}

The samples of the fungal biomass of Blakeslea trispora were taken for the comparative analyses using the spectrophotometer and HPLC (Fig. 3). The results of the measurements are presented in Table 3.

The prediction quality of the UV-vis method is sufficient and the obtained results are very close to the ones, being measured with the HPLC technique. The differences between the measurements were not statistically significant.

As a conclusion, the proposed method is promising for both routine industrial work and academic research, providing the rapid analysis for simultaneous measurements of lycopene and $\beta$-carotene with minimal personnel training and costly technique for immediate predictions.

The elaborated method can be used for simultaneous measurement of lycopene and $\beta$-carotene in any carotenoid source, i.e. plants, fungi, algae, tissues etc, where the concentrations of these carotenoids are prevailed. The method can be also used for controlling food and/ or dietary supplements which contain lycopene and $\beta$-carotene.

\section{REFERENCES}

Baranska M, Schütze W, Schulz H (2006) Determination of lycopene and $\beta$-carotene content in tomato fruits and related products: comparison of FT-Raman, ATR-IR, and NIR spectroscopy. Anal Chem 78: $8456-8461$.

Britton G, Liaaen-Jensen S, Pfander Carotenoids H (2004) Carotenoids Handbook. Birkhäuser Verlag - Basel; Boston; Berlin.

B-carotene from Blakeslea trispora (2003) FNP 52, Add. 11. Prepared at the 61st JECF $A$.

Choudhari SM, Ananthanarayan L, Singhal RS (2008) Use of metabolic stimulators and inhibitors for enhanced production of $\beta$-carotene and lycopene by Blakeslea trispora NRRL 2895 and 2896. Bioresource Technol 99: 3166-3173.

Cserhati T, Forgacs E, Morais MH, Mota T (2000) Liquid chromatography of natural pigments. Biomed Chromatography 14: 281-286.

Echavarri-Erasun C, Johnson EA (2002) Fungal carotenoids. Appl Mycology Biotech 2: 45-85.

Emenhiser C, Simonovic N, Sander LC, Schwartz SJ (1996) Separation of geometrical carotenoid isomers in biological extracts using a polymeric $\mathrm{C}_{30}$ column in reversed-phase liquid chromatography. I Agric Food Chem 44: 3887-3893.

Ermakov IV, Ermakova MR, Gellermann W, Lademann J (2004) Noninvasive selective detection of lycopene and $\beta$-carotene in human skin using Raman spectroscopy. J Biomed Optics 9: 332-338.

Lycopene from Blakeslea trispora (2006) FAO JECFA Monographs 3. Prepared at the $67^{\text {th }} \mathrm{JECF} A$.

Mantzouridou F, Roukas T, Kotzekidou P, Liakopoulou M (2002) Optimization of $\beta$-carotene production from synthetic medium by Blakeslea trispora: a mathematical modeling. Appl Biochem Eng 101: $153-175$.

Mantzouridou F, Tsimidou MZ (2007) On the monitoring of carotenogenesis by Blakeslea trispora using HPLC. Food Chem 104: 439-444.

Mehta BJ, Cerda-Olmedo E (1995) Mutants of carotene production in Blakeslea trispora. Appl Microbiol Biotechnol 42: 836-838.

Mehta BJ, Cerda-Olmedo E (1999) Lycopene cyclization in Blakeslea trispora. Mycoscience 40: 307-310.

Mehta BJ, Obraztsova IN, Cerda-Olmedo E (2003) Mutants and intersexual heterokaryons of Blakeslea trispora for production of 3-carotene and lycopene. Appl Environ Microbiol 69: 4043-4048.

Nardo De T, Shiroma-Kian C, Halim Y, Francis D, Rodrigues-Saona LE (2009) Rapid and simultaneous determination of lycopene and $\beta$-carotene contents in tomato juice by infrared spectroscopy. $J \mathrm{Ag}$ ric Food Chem 57: 1105-1112.

Oliver J, Palou A (2000) Chromatographic determination of carotenoids in foods. J Chromatography 881: 543-555.

Patent of Ukraine No. 83420 (2008) Method of manufacturing of lycopene biomass. Filed Oct. 4, 2006; Publ. Jul. 10, 2008, Bull. No. 13. (in Ukrainian)

Pedro AMK, Ferreira MMC (2005) Nondestructive determination of solids and carotenoids in tomato products by near-infrared spectroscopy and multivariate calibration. Anal Chem 77: 2505-2511.

Pegklidou K, Mantzouridou F, Tsimidou MZ (2008) Lycopene production using Blakeslea trispora in the presence of 2-methyl imidazole: yield, selectivity, and safety aspects. I Agric Food Chem 56: 44824490 .

Peshkova VM, Gromova MI (1965) Practical Guide on Spectrophotometry and Colorimetry. $2^{\text {nd }}$ edition. Publisher: Moscow University (in Russian).

STATISTICA@’99 (1999) Edition. Copyright ${ }^{\circledR}$ 1984-1999 by StatSoft, Inc.

Xu F, Yuan QP, Dong HR (2006) Determination of lycopene and $\beta$-carotene by high-performance liquid chromatography using sudan I as internal standard. J Chromatography 838: 44-49. 